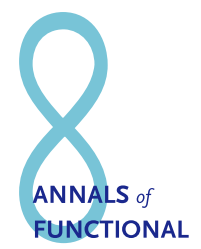

Ann. Funct. Anal. 7 (2016), no. 4, 609-621

http://dx.doi.org/10.1215/20088752-3660801

ISSN: 2008-8752 (electronic)

http://projecteuclid.org/afa

ANALYSIS

\title{
ON $m$-GENERALIZED INVERTIBLE OPERATORS ON BANACH SPACES
}

\author{
HAMID EZZAHRAOUI
}

Communicated by M. Mbekhta

Abstract. A bounded linear operator $S$ on a Banach space $X$ is called an $m$-left generalized inverse of an operator $T$ for a positive integer $m$ if

$$
T \sum_{j=0}^{m}(-1)^{j}\left(\begin{array}{c}
m \\
j
\end{array}\right) S^{m-j} T^{m-j}=0,
$$

and it is called an m-right generalized inverse of $T$ if

$$
S \sum_{j=0}^{m}(-1)^{j}\left(\begin{array}{c}
m \\
j
\end{array}\right) T^{m-j} S^{m-j}=0 .
$$

If $T$ is both an $m$-left and an $m$-right generalized inverse of $T$, then it is said to be an $m$-generalized inverse of $T$.

This paper has two purposes. The first is to extend the notion of generalized inverse to $m$-generalized inverse of an operator on Banach spaces and to give some structure results. The second is to generalize some properties of $m$-partial isometries on Hilbert spaces to the class of $m$-left generalized invertible operators on Banach spaces. In particular, we study some cases in which a power of an $m$-left generalized invertible operator is again $m$-left generalized invertible.

\section{INTRODUCTION AND PRELIMINARIES}

Throughout this paper, $X$ shall denote a complex Banach space, and $\mathcal{L}(X)$ shall denote the algebra of all bounded linear operators on $X$. We denote $X$ by

Copyright 2016 by the Tusi Mathematical Research Group.

Received Mar. 13, 2016; Accepted May 3, 2016.

2010 Mathematics Subject Classification. Primary 47B48; Secondary 47B99.

Keywords. $m$-isometry, $m$-partial-isometry, $m$-left inverse, $m$-right inverse, $m$-left generalized inverse, $m$-right generalized inverse. 


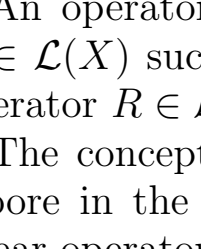

Ann. Funct. Anal. 7 (2016), no. 4, 609-621

http://dx.doi.org/10.1215/20088752-3660801

ISSN: 2008-8752 (electronic)

http://projecteuclid.org/afa

ANALYSIS

\title{
ON $m$-GENERALIZED INVERTIBLE OPERATORS ON BANACH SPACES
}

\author{
HAMID EZZAHRAOUI
}

Communicated by M. Mbekhta

Abstract. A bounded linear operator $S$ on a Banach space $X$ is called an $m$-left generalized inverse of an operator $T$ for a positive integer $m$ if

$$
T \sum_{j=0}^{m}(-1)^{j}\left(\begin{array}{c}
m \\
j
\end{array}\right) S^{m-j} T^{m-j}=0,
$$

and it is called an m-right generalized inverse of $T$ if

$$
S \sum_{j=0}^{m}(-1)^{j}\left(\begin{array}{c}
m \\
j
\end{array}\right) T^{m-j} S^{m-j}=0 .
$$

If $T$ is both an $m$-left and an $m$-right generalized inverse of $T$, then it is said to be an $m$-generalized inverse of $T$.

This paper has two purposes. The first is to extend the notion of generalized inverse to $m$-generalized inverse of an operator on Banach spaces and to give some structure results. The second is to generalize some properties of $m$-partial isometries on Hilbert spaces to the class of $m$-left generalized invertible operators on Banach spaces. In particular, we study some cases in which a power of an $m$-left generalized invertible operator is again $m$-left generalized invertible.

\section{INTRODUCTION AND PRELIMINARIES}

Throughout this paper, $X$ shall denote a complex Banach space, and $\mathcal{L}(X)$ shall denote the algebra of all bounded linear operators on $X$. We denote $X$ by

Copyright 2016 by the Tusi Mathematical Research Group.

Received Mar. 13, 2016; Accepted May 3, 2016.

2010 Mathematics Subject Classification. Primary 47B48; Secondary 47B99.

Keywords. $m$-isometry, $m$-partial-isometry, $m$-left inverse, $m$-right inverse, $m$-left generalized inverse, $m$-right generalized inverse. 


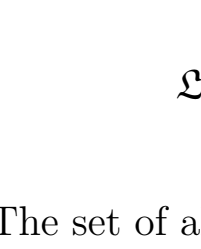

Ann. Funct. Anal. 7 (2016), no. 4, 609-621

http://dx.doi.org/10.1215/20088752-3660801

ISSN: 2008-8752 (electronic)

http://projecteuclid.org/afa

ANALYSIS

\title{
ON $m$-GENERALIZED INVERTIBLE OPERATORS ON BANACH SPACES
}

\author{
HAMID EZZAHRAOUI
}

Communicated by M. Mbekhta

Abstract. A bounded linear operator $S$ on a Banach space $X$ is called an $m$-left generalized inverse of an operator $T$ for a positive integer $m$ if

$$
T \sum_{j=0}^{m}(-1)^{j}\left(\begin{array}{c}
m \\
j
\end{array}\right) S^{m-j} T^{m-j}=0,
$$

and it is called an m-right generalized inverse of $T$ if

$$
S \sum_{j=0}^{m}(-1)^{j}\left(\begin{array}{c}
m \\
j
\end{array}\right) T^{m-j} S^{m-j}=0 .
$$

If $T$ is both an $m$-left and an $m$-right generalized inverse of $T$, then it is said to be an $m$-generalized inverse of $T$.

This paper has two purposes. The first is to extend the notion of generalized inverse to $m$-generalized inverse of an operator on Banach spaces and to give some structure results. The second is to generalize some properties of $m$-partial isometries on Hilbert spaces to the class of $m$-left generalized invertible operators on Banach spaces. In particular, we study some cases in which a power of an $m$-left generalized invertible operator is again $m$-left generalized invertible.

\section{INTRODUCTION AND PRELIMINARIES}

Throughout this paper, $X$ shall denote a complex Banach space, and $\mathcal{L}(X)$ shall denote the algebra of all bounded linear operators on $X$. We denote $X$ by

Copyright 2016 by the Tusi Mathematical Research Group.

Received Mar. 13, 2016; Accepted May 3, 2016.

2010 Mathematics Subject Classification. Primary 47B48; Secondary 47B99.

Keywords. $m$-isometry, $m$-partial-isometry, $m$-left inverse, $m$-right inverse, $m$-left generalized inverse, $m$-right generalized inverse. 


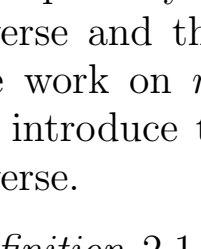

Ann. Funct. Anal. 7 (2016), no. 4, 609-621

http://dx.doi.org/10.1215/20088752-3660801

ISSN: 2008-8752 (electronic)

http://projecteuclid.org/afa

ANALYSIS

\title{
ON $m$-GENERALIZED INVERTIBLE OPERATORS ON BANACH SPACES
}

\author{
HAMID EZZAHRAOUI
}

Communicated by M. Mbekhta

Abstract. A bounded linear operator $S$ on a Banach space $X$ is called an $m$-left generalized inverse of an operator $T$ for a positive integer $m$ if

$$
T \sum_{j=0}^{m}(-1)^{j}\left(\begin{array}{c}
m \\
j
\end{array}\right) S^{m-j} T^{m-j}=0,
$$

and it is called an m-right generalized inverse of $T$ if

$$
S \sum_{j=0}^{m}(-1)^{j}\left(\begin{array}{c}
m \\
j
\end{array}\right) T^{m-j} S^{m-j}=0 .
$$

If $T$ is both an $m$-left and an $m$-right generalized inverse of $T$, then it is said to be an $m$-generalized inverse of $T$.

This paper has two purposes. The first is to extend the notion of generalized inverse to $m$-generalized inverse of an operator on Banach spaces and to give some structure results. The second is to generalize some properties of $m$-partial isometries on Hilbert spaces to the class of $m$-left generalized invertible operators on Banach spaces. In particular, we study some cases in which a power of an $m$-left generalized invertible operator is again $m$-left generalized invertible.

\section{INTRODUCTION AND PRELIMINARIES}

Throughout this paper, $X$ shall denote a complex Banach space, and $\mathcal{L}(X)$ shall denote the algebra of all bounded linear operators on $X$. We denote $X$ by

Copyright 2016 by the Tusi Mathematical Research Group.

Received Mar. 13, 2016; Accepted May 3, 2016.

2010 Mathematics Subject Classification. Primary 47B48; Secondary 47B99.

Keywords. $m$-isometry, $m$-partial-isometry, $m$-left inverse, $m$-right inverse, $m$-left generalized inverse, $m$-right generalized inverse. 


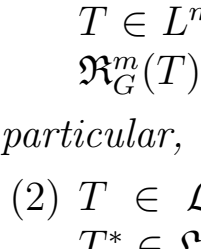

Ann. Funct. Anal. 7 (2016), no. 4, 609-621

http://dx.doi.org/10.1215/20088752-3660801

ISSN: 2008-8752 (electronic)

http://projecteuclid.org/afa

ANALYSIS

\title{
ON $m$-GENERALIZED INVERTIBLE OPERATORS ON BANACH SPACES
}

\author{
HAMID EZZAHRAOUI
}

Communicated by M. Mbekhta

Abstract. A bounded linear operator $S$ on a Banach space $X$ is called an $m$-left generalized inverse of an operator $T$ for a positive integer $m$ if

$$
T \sum_{j=0}^{m}(-1)^{j}\left(\begin{array}{c}
m \\
j
\end{array}\right) S^{m-j} T^{m-j}=0,
$$

and it is called an m-right generalized inverse of $T$ if

$$
S \sum_{j=0}^{m}(-1)^{j}\left(\begin{array}{c}
m \\
j
\end{array}\right) T^{m-j} S^{m-j}=0 .
$$

If $T$ is both an $m$-left and an $m$-right generalized inverse of $T$, then it is said to be an $m$-generalized inverse of $T$.

This paper has two purposes. The first is to extend the notion of generalized inverse to $m$-generalized inverse of an operator on Banach spaces and to give some structure results. The second is to generalize some properties of $m$-partial isometries on Hilbert spaces to the class of $m$-left generalized invertible operators on Banach spaces. In particular, we study some cases in which a power of an $m$-left generalized invertible operator is again $m$-left generalized invertible.

\section{INTRODUCTION AND PRELIMINARIES}

Throughout this paper, $X$ shall denote a complex Banach space, and $\mathcal{L}(X)$ shall denote the algebra of all bounded linear operators on $X$. We denote $X$ by

Copyright 2016 by the Tusi Mathematical Research Group.

Received Mar. 13, 2016; Accepted May 3, 2016.

2010 Mathematics Subject Classification. Primary 47B48; Secondary 47B99.

Keywords. $m$-isometry, $m$-partial-isometry, $m$-left inverse, $m$-right inverse, $m$-left generalized inverse, $m$-right generalized inverse. 


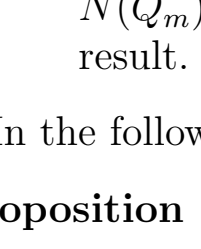

Ann. Funct. Anal. 7 (2016), no. 4, 609-621

http://dx.doi.org/10.1215/20088752-3660801

ISSN: 2008-8752 (electronic)

http://projecteuclid.org/afa

ANALYSIS

\title{
ON $m$-GENERALIZED INVERTIBLE OPERATORS ON BANACH SPACES
}

\author{
HAMID EZZAHRAOUI
}

Communicated by M. Mbekhta

Abstract. A bounded linear operator $S$ on a Banach space $X$ is called an $m$-left generalized inverse of an operator $T$ for a positive integer $m$ if

$$
T \sum_{j=0}^{m}(-1)^{j}\left(\begin{array}{c}
m \\
j
\end{array}\right) S^{m-j} T^{m-j}=0,
$$

and it is called an m-right generalized inverse of $T$ if

$$
S \sum_{j=0}^{m}(-1)^{j}\left(\begin{array}{c}
m \\
j
\end{array}\right) T^{m-j} S^{m-j}=0 .
$$

If $T$ is both an $m$-left and an $m$-right generalized inverse of $T$, then it is said to be an $m$-generalized inverse of $T$.

This paper has two purposes. The first is to extend the notion of generalized inverse to $m$-generalized inverse of an operator on Banach spaces and to give some structure results. The second is to generalize some properties of $m$-partial isometries on Hilbert spaces to the class of $m$-left generalized invertible operators on Banach spaces. In particular, we study some cases in which a power of an $m$-left generalized invertible operator is again $m$-left generalized invertible.

\section{INTRODUCTION AND PRELIMINARIES}

Throughout this paper, $X$ shall denote a complex Banach space, and $\mathcal{L}(X)$ shall denote the algebra of all bounded linear operators on $X$. We denote $X$ by

Copyright 2016 by the Tusi Mathematical Research Group.

Received Mar. 13, 2016; Accepted May 3, 2016.

2010 Mathematics Subject Classification. Primary 47B48; Secondary 47B99.

Keywords. $m$-isometry, $m$-partial-isometry, $m$-left inverse, $m$-right inverse, $m$-left generalized inverse, $m$-right generalized inverse. 


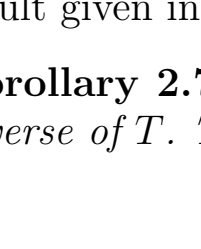

Ann. Funct. Anal. 7 (2016), no. 4, 609-621

http://dx.doi.org/10.1215/20088752-3660801

ISSN: 2008-8752 (electronic)

http://projecteuclid.org/afa

ANALYSIS

\title{
ON $m$-GENERALIZED INVERTIBLE OPERATORS ON BANACH SPACES
}

\author{
HAMID EZZAHRAOUI
}

Communicated by M. Mbekhta

Abstract. A bounded linear operator $S$ on a Banach space $X$ is called an $m$-left generalized inverse of an operator $T$ for a positive integer $m$ if

$$
T \sum_{j=0}^{m}(-1)^{j}\left(\begin{array}{c}
m \\
j
\end{array}\right) S^{m-j} T^{m-j}=0,
$$

and it is called an m-right generalized inverse of $T$ if

$$
S \sum_{j=0}^{m}(-1)^{j}\left(\begin{array}{c}
m \\
j
\end{array}\right) T^{m-j} S^{m-j}=0 .
$$

If $T$ is both an $m$-left and an $m$-right generalized inverse of $T$, then it is said to be an $m$-generalized inverse of $T$.

This paper has two purposes. The first is to extend the notion of generalized inverse to $m$-generalized inverse of an operator on Banach spaces and to give some structure results. The second is to generalize some properties of $m$-partial isometries on Hilbert spaces to the class of $m$-left generalized invertible operators on Banach spaces. In particular, we study some cases in which a power of an $m$-left generalized invertible operator is again $m$-left generalized invertible.

\section{INTRODUCTION AND PRELIMINARIES}

Throughout this paper, $X$ shall denote a complex Banach space, and $\mathcal{L}(X)$ shall denote the algebra of all bounded linear operators on $X$. We denote $X$ by

Copyright 2016 by the Tusi Mathematical Research Group.

Received Mar. 13, 2016; Accepted May 3, 2016.

2010 Mathematics Subject Classification. Primary 47B48; Secondary 47B99.

Keywords. $m$-isometry, $m$-partial-isometry, $m$-left inverse, $m$-right inverse, $m$-left generalized inverse, $m$-right generalized inverse. 


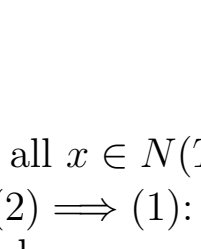

Ann. Funct. Anal. 7 (2016), no. 4, 609-621

http://dx.doi.org/10.1215/20088752-3660801

ISSN: 2008-8752 (electronic)

http://projecteuclid.org/afa

ANALYSIS

\title{
ON $m$-GENERALIZED INVERTIBLE OPERATORS ON BANACH SPACES
}

\author{
HAMID EZZAHRAOUI
}

Communicated by M. Mbekhta

Abstract. A bounded linear operator $S$ on a Banach space $X$ is called an $m$-left generalized inverse of an operator $T$ for a positive integer $m$ if

$$
T \sum_{j=0}^{m}(-1)^{j}\left(\begin{array}{c}
m \\
j
\end{array}\right) S^{m-j} T^{m-j}=0,
$$

and it is called an m-right generalized inverse of $T$ if

$$
S \sum_{j=0}^{m}(-1)^{j}\left(\begin{array}{c}
m \\
j
\end{array}\right) T^{m-j} S^{m-j}=0 .
$$

If $T$ is both an $m$-left and an $m$-right generalized inverse of $T$, then it is said to be an $m$-generalized inverse of $T$.

This paper has two purposes. The first is to extend the notion of generalized inverse to $m$-generalized inverse of an operator on Banach spaces and to give some structure results. The second is to generalize some properties of $m$-partial isometries on Hilbert spaces to the class of $m$-left generalized invertible operators on Banach spaces. In particular, we study some cases in which a power of an $m$-left generalized invertible operator is again $m$-left generalized invertible.

\section{INTRODUCTION AND PRELIMINARIES}

Throughout this paper, $X$ shall denote a complex Banach space, and $\mathcal{L}(X)$ shall denote the algebra of all bounded linear operators on $X$. We denote $X$ by

Copyright 2016 by the Tusi Mathematical Research Group.

Received Mar. 13, 2016; Accepted May 3, 2016.

2010 Mathematics Subject Classification. Primary 47B48; Secondary 47B99.

Keywords. $m$-isometry, $m$-partial-isometry, $m$-left inverse, $m$-right inverse, $m$-left generalized inverse, $m$-right generalized inverse. 


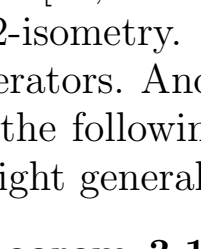

Ann. Funct. Anal. 7 (2016), no. 4, 609-621

http://dx.doi.org/10.1215/20088752-3660801

ISSN: 2008-8752 (electronic)

http://projecteuclid.org/afa

ANALYSIS

\title{
ON $m$-GENERALIZED INVERTIBLE OPERATORS ON BANACH SPACES
}

\author{
HAMID EZZAHRAOUI
}

Communicated by M. Mbekhta

Abstract. A bounded linear operator $S$ on a Banach space $X$ is called an $m$-left generalized inverse of an operator $T$ for a positive integer $m$ if

$$
T \sum_{j=0}^{m}(-1)^{j}\left(\begin{array}{c}
m \\
j
\end{array}\right) S^{m-j} T^{m-j}=0,
$$

and it is called an m-right generalized inverse of $T$ if

$$
S \sum_{j=0}^{m}(-1)^{j}\left(\begin{array}{c}
m \\
j
\end{array}\right) T^{m-j} S^{m-j}=0 .
$$

If $T$ is both an $m$-left and an $m$-right generalized inverse of $T$, then it is said to be an $m$-generalized inverse of $T$.

This paper has two purposes. The first is to extend the notion of generalized inverse to $m$-generalized inverse of an operator on Banach spaces and to give some structure results. The second is to generalize some properties of $m$-partial isometries on Hilbert spaces to the class of $m$-left generalized invertible operators on Banach spaces. In particular, we study some cases in which a power of an $m$-left generalized invertible operator is again $m$-left generalized invertible.

\section{INTRODUCTION AND PRELIMINARIES}

Throughout this paper, $X$ shall denote a complex Banach space, and $\mathcal{L}(X)$ shall denote the algebra of all bounded linear operators on $X$. We denote $X$ by

Copyright 2016 by the Tusi Mathematical Research Group.

Received Mar. 13, 2016; Accepted May 3, 2016.

2010 Mathematics Subject Classification. Primary 47B48; Secondary 47B99.

Keywords. $m$-isometry, $m$-partial-isometry, $m$-left inverse, $m$-right inverse, $m$-left generalized inverse, $m$-right generalized inverse. 


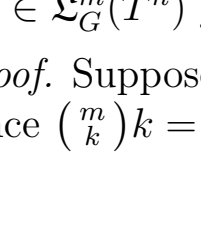

Ann. Funct. Anal. 7 (2016), no. 4, 609-621

http://dx.doi.org/10.1215/20088752-3660801

ISSN: 2008-8752 (electronic)

http://projecteuclid.org/afa

ANALYSIS

\title{
ON $m$-GENERALIZED INVERTIBLE OPERATORS ON BANACH SPACES
}

\author{
HAMID EZZAHRAOUI
}

Communicated by M. Mbekhta

Abstract. A bounded linear operator $S$ on a Banach space $X$ is called an $m$-left generalized inverse of an operator $T$ for a positive integer $m$ if

$$
T \sum_{j=0}^{m}(-1)^{j}\left(\begin{array}{c}
m \\
j
\end{array}\right) S^{m-j} T^{m-j}=0,
$$

and it is called an m-right generalized inverse of $T$ if

$$
S \sum_{j=0}^{m}(-1)^{j}\left(\begin{array}{c}
m \\
j
\end{array}\right) T^{m-j} S^{m-j}=0 .
$$

If $T$ is both an $m$-left and an $m$-right generalized inverse of $T$, then it is said to be an $m$-generalized inverse of $T$.

This paper has two purposes. The first is to extend the notion of generalized inverse to $m$-generalized inverse of an operator on Banach spaces and to give some structure results. The second is to generalize some properties of $m$-partial isometries on Hilbert spaces to the class of $m$-left generalized invertible operators on Banach spaces. In particular, we study some cases in which a power of an $m$-left generalized invertible operator is again $m$-left generalized invertible.

\section{INTRODUCTION AND PRELIMINARIES}

Throughout this paper, $X$ shall denote a complex Banach space, and $\mathcal{L}(X)$ shall denote the algebra of all bounded linear operators on $X$. We denote $X$ by

Copyright 2016 by the Tusi Mathematical Research Group.

Received Mar. 13, 2016; Accepted May 3, 2016.

2010 Mathematics Subject Classification. Primary 47B48; Secondary 47B99.

Keywords. $m$-isometry, $m$-partial-isometry, $m$-left inverse, $m$-right inverse, $m$-left generalized inverse, $m$-right generalized inverse. 


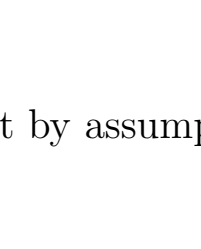

Ann. Funct. Anal. 7 (2016), no. 4, 609-621

http://dx.doi.org/10.1215/20088752-3660801

ISSN: 2008-8752 (electronic)

http://projecteuclid.org/afa

ANALYSIS

\title{
ON $m$-GENERALIZED INVERTIBLE OPERATORS ON BANACH SPACES
}

\author{
HAMID EZZAHRAOUI
}

Communicated by M. Mbekhta

Abstract. A bounded linear operator $S$ on a Banach space $X$ is called an $m$-left generalized inverse of an operator $T$ for a positive integer $m$ if

$$
T \sum_{j=0}^{m}(-1)^{j}\left(\begin{array}{c}
m \\
j
\end{array}\right) S^{m-j} T^{m-j}=0,
$$

and it is called an m-right generalized inverse of $T$ if

$$
S \sum_{j=0}^{m}(-1)^{j}\left(\begin{array}{c}
m \\
j
\end{array}\right) T^{m-j} S^{m-j}=0 .
$$

If $T$ is both an $m$-left and an $m$-right generalized inverse of $T$, then it is said to be an $m$-generalized inverse of $T$.

This paper has two purposes. The first is to extend the notion of generalized inverse to $m$-generalized inverse of an operator on Banach spaces and to give some structure results. The second is to generalize some properties of $m$-partial isometries on Hilbert spaces to the class of $m$-left generalized invertible operators on Banach spaces. In particular, we study some cases in which a power of an $m$-left generalized invertible operator is again $m$-left generalized invertible.

\section{INTRODUCTION AND PRELIMINARIES}

Throughout this paper, $X$ shall denote a complex Banach space, and $\mathcal{L}(X)$ shall denote the algebra of all bounded linear operators on $X$. We denote $X$ by

Copyright 2016 by the Tusi Mathematical Research Group.

Received Mar. 13, 2016; Accepted May 3, 2016.

2010 Mathematics Subject Classification. Primary 47B48; Secondary 47B99.

Keywords. $m$-isometry, $m$-partial-isometry, $m$-left inverse, $m$-right inverse, $m$-left generalized inverse, $m$-right generalized inverse. 


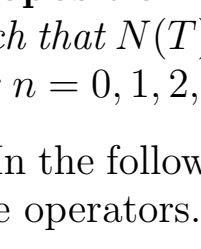

Ann. Funct. Anal. 7 (2016), no. 4, 609-621

http://dx.doi.org/10.1215/20088752-3660801

ISSN: 2008-8752 (electronic)

http://projecteuclid.org/afa

ANALYSIS

\title{
ON $m$-GENERALIZED INVERTIBLE OPERATORS ON BANACH SPACES
}

\author{
HAMID EZZAHRAOUI
}

Communicated by M. Mbekhta

Abstract. A bounded linear operator $S$ on a Banach space $X$ is called an $m$-left generalized inverse of an operator $T$ for a positive integer $m$ if

$$
T \sum_{j=0}^{m}(-1)^{j}\left(\begin{array}{c}
m \\
j
\end{array}\right) S^{m-j} T^{m-j}=0,
$$

and it is called an m-right generalized inverse of $T$ if

$$
S \sum_{j=0}^{m}(-1)^{j}\left(\begin{array}{c}
m \\
j
\end{array}\right) T^{m-j} S^{m-j}=0 .
$$

If $T$ is both an $m$-left and an $m$-right generalized inverse of $T$, then it is said to be an $m$-generalized inverse of $T$.

This paper has two purposes. The first is to extend the notion of generalized inverse to $m$-generalized inverse of an operator on Banach spaces and to give some structure results. The second is to generalize some properties of $m$-partial isometries on Hilbert spaces to the class of $m$-left generalized invertible operators on Banach spaces. In particular, we study some cases in which a power of an $m$-left generalized invertible operator is again $m$-left generalized invertible.

\section{INTRODUCTION AND PRELIMINARIES}

Throughout this paper, $X$ shall denote a complex Banach space, and $\mathcal{L}(X)$ shall denote the algebra of all bounded linear operators on $X$. We denote $X$ by

Copyright 2016 by the Tusi Mathematical Research Group.

Received Mar. 13, 2016; Accepted May 3, 2016.

2010 Mathematics Subject Classification. Primary 47B48; Secondary 47B99.

Keywords. $m$-isometry, $m$-partial-isometry, $m$-left inverse, $m$-right inverse, $m$-left generalized inverse, $m$-right generalized inverse. 


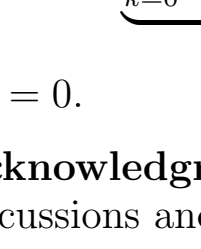

Ann. Funct. Anal. 7 (2016), no. 4, 609-621

http://dx.doi.org/10.1215/20088752-3660801

ISSN: 2008-8752 (electronic)

http://projecteuclid.org/afa

ANALYSIS

\title{
ON $m$-GENERALIZED INVERTIBLE OPERATORS ON BANACH SPACES
}

\author{
HAMID EZZAHRAOUI
}

Communicated by M. Mbekhta

Abstract. A bounded linear operator $S$ on a Banach space $X$ is called an $m$-left generalized inverse of an operator $T$ for a positive integer $m$ if

$$
T \sum_{j=0}^{m}(-1)^{j}\left(\begin{array}{c}
m \\
j
\end{array}\right) S^{m-j} T^{m-j}=0,
$$

and it is called an m-right generalized inverse of $T$ if

$$
S \sum_{j=0}^{m}(-1)^{j}\left(\begin{array}{c}
m \\
j
\end{array}\right) T^{m-j} S^{m-j}=0 .
$$

If $T$ is both an $m$-left and an $m$-right generalized inverse of $T$, then it is said to be an $m$-generalized inverse of $T$.

This paper has two purposes. The first is to extend the notion of generalized inverse to $m$-generalized inverse of an operator on Banach spaces and to give some structure results. The second is to generalize some properties of $m$-partial isometries on Hilbert spaces to the class of $m$-left generalized invertible operators on Banach spaces. In particular, we study some cases in which a power of an $m$-left generalized invertible operator is again $m$-left generalized invertible.

\section{INTRODUCTION AND PRELIMINARIES}

Throughout this paper, $X$ shall denote a complex Banach space, and $\mathcal{L}(X)$ shall denote the algebra of all bounded linear operators on $X$. We denote $X$ by

Copyright 2016 by the Tusi Mathematical Research Group.

Received Mar. 13, 2016; Accepted May 3, 2016.

2010 Mathematics Subject Classification. Primary 47B48; Secondary 47B99.

Keywords. $m$-isometry, $m$-partial-isometry, $m$-left inverse, $m$-right inverse, $m$-left generalized inverse, $m$-right generalized inverse. 